\title{
Observation of Pseudo Plume Behavior by Hydrate Sedimentary Layer Decomposition
}

\author{
Yusuke TAKahashi, Ryosuke Ezure, Shun TAKano, Hiroyuki Komatsu, Kazuaki Yamagiwa, and Hideo TAJIMA* \\ Graduate School of Science and Technology, Niigata University, 2-8050 Ikarashi, Niigata 950-2181, Japan
}

\begin{abstract}
We are focusing on the practical use of methane hydrate. For recovery and use of it as an energy resource, it is necessary to consider the possibility of clogging in the recovery pipe due to the rehydration of bubbles. The purpose of this research was to observe experimentally and evaluate theoretically the decomposition behavior of hydrate sedimentary layer and the rising behavior of bubbles generated by hydrate decomposition. Chlorodifluoromethane was used as a low pressure model gas of methane. Hydrate sedimentary layer was produced by cooling and pressurizing water in countercurrent contact with gas using a hydrate formation recovery device. The recovered hydrate was decomposed by the heating or depressurization method, without flowing water. Two theoretical rising velocities were derived from the theoretical value with using the Navier-Stokes equation or the values in consideration of the bubble shape and hydrate film existence. The experimental rising velocities of small spherical bubbles radius agreed well with the theoretical value by the Navier-Stokes equation. The relatively large elliptical bubbles showed a behavior close to the theoretical value of bubble with hydrate film. Under the pressure and temperature conditions closer to the hydrate equilibrium line, almost no generated bubbles could be identified visually.
\end{abstract}

\section{Introduction}

Development of alternative energy, improvement of power generation method, and energy saving of equipment are required as a solution approach to the energy problem of fossil fuel exhaustion. We are focusing on the practical use of methane hydrate, which is expected to be an alternative energy source to reduce $\mathrm{CO}_{2}$ emission in the future. Unlike shale gas and coalbed methane, methane hydrate reservoir characterization and gas production methods are still at the research stage. If methane hydrate can be used as an energy resource, it will contribute to energy security by securing domestic resources, use as conventional alternative energy, and carbon dioxide emission reduction in combustion compared with petroleum and coal as natural gas. Gas hydrate is an ice-like solid crystal in which guest molecules are incorporated in a cage-like space composed of hydrogen bonds of water molecules. Methane hydrate can store 160 to $170 \mathrm{~m}^{3}$ of methane gas per $1 \mathrm{~m}^{3}$ (Kataoka et al., 2007). Natural methane hydrates form at low temperatures and high pressure, and gas hydrates can exist in a stable under the conditions of appropriate temperature and pressure such as deep sea floor. Methane hydrates that exist in the waters near Japan are roughly classified into two types (Sato et al., 2016): sand-type methane hydrate and surficial and shallow methane hydrate. When these two methane hydrates are compared from the viewpoint of recovery, it is thought that surficial and shallow hydrates present on shallow seabed surfaces are easier to recover than sand-layer type methane hydrates present in deep seabed. The surficial and shallow methane hydrate presented under the seafloor generates bubble groups (that is "plume") by decomposition. As an example of practical use of surface type methane hydrate recovery, a recovery method using a dome-like film is being studied (Aoyama et al., 2017). When bubble groups are collected by using a channel pipe, it is necessary to consider the possibility of clogging in the recovery pipe due to the rehydration of bubbles. It is important for efficient gas recovery to understand the generation and rising movement behaviors of the bubble group. The purpose of this research is to observe experimentally and evaluate theoretically the decomposition behavior of hydrate sedimentary layer and the rising behavior of bubbles generated by hydrate decomposition.

\section{Experimental Method}

\subsection{Experimental equipment}

All experiments were carried out using the hydrate-based gas recovery equipment as shown in Figure 1. This equipment uses two low-temperature thermostats on the gas hydrate production side (LTI-1200 EYELA Co. Ltd) and the gas hydrate decomposition side (FMU-2631 Fukushima Co. Ltd) to cool the entire reactor to a

*Corresponding Author:h_tajima@eng.niigata-u.ac.jp 
constant temperature. Kenics-type mixing elements of SUS316 static mixer (Noritake Co. Ltd., Japan, 24 elements, $400 \mathrm{~mm}$ length, pitch 1.5 ) as the flow reactor was inserted into a glass tube (i.d. $11.0 \mathrm{~mm}$ ). The inlet gas flow rate was controlled by a mass flow controller (MFC) and the outlet gas flow rate was measured by a mass flow meter (MFM) (KOFLOC). Experimental pressure in the reactor was controlled by a back pressure valve (AP Tech, BP1000 series) and measured by a pressure transducer (Nagano, KH-15). The experimental temperature was controlled by thermostatic each chamber and measured by resistance temperature detectors. Pressure, temperature, and the outlet gas flow rate were recorded on a data logger system (KEYENCE) and collected data with a PC. The formed hydrate slurry was collected in a glass hydrate sedimentary vessel (volume $500 \mathrm{~mL}$ ) installed in the decomposition-side thermostat by flowing it using a pump (EYELA)

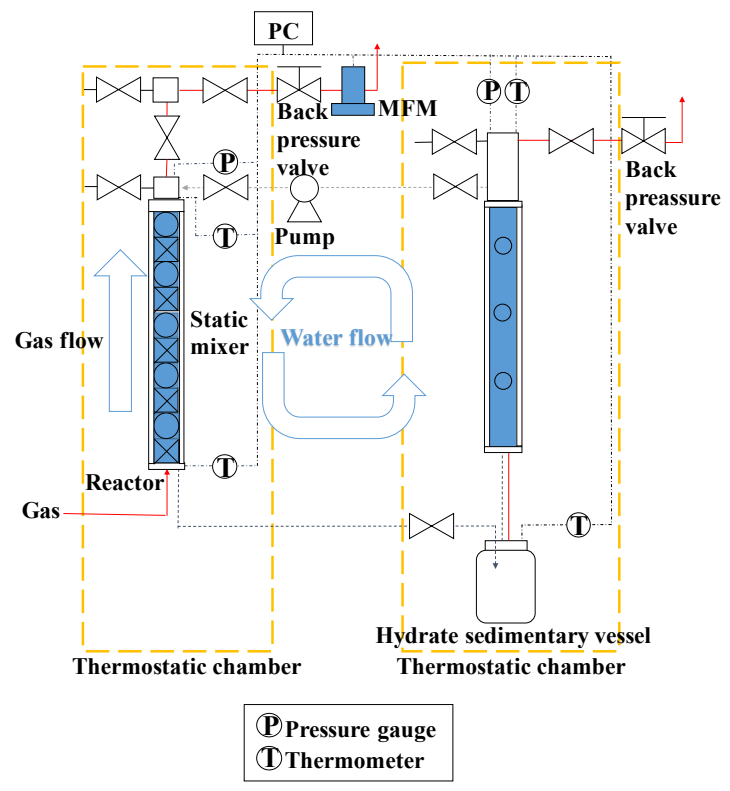

Figure 1. Experimental equipment

\subsection{Experimental condition}

Chlorodifluoromethane $\left(\mathrm{CHClF}_{2}, \mathrm{R} 22\right)$ was used as a low pressure model gas of methane. Deionized water was used for hydrate formation. The flow rate of gas and water is about $200 \mathrm{ml} / \mathrm{min}$ and $138 \mathrm{ml} / \mathrm{min}$, respectively. Pressure and temperature in the reactor were set at 0.300 $\mathrm{MPa} \quad( \pm 0.005 \mathrm{MPa})$ and $278-280 \quad \mathrm{~K} \quad( \pm 0.5 \quad \mathrm{~K})$, respectively. After hydrate formation and sedimentation, hydrate decomposition was carried out at various pressure and temperature in the decomposition-side thermostatic chamber.

\subsection{Experimental procedure}

\subsubsection{Hydrate formation}

Deionized water was filled in the reactor, hydrate sedimentary vessel, and pipe for water circulation and cooled until the desired temperature. During cooling, the water supplying pump was operated to circulate the water. The R22 gas was then injected to the reactor at the desired pressure, and the R22 gas and water were countercurrently contacted to form hydrate. When the outlet gas flow rate became constant before hydrate formation, water in the equipment was assumed to be saturated with R22 gas.

\subsubsection{Hydrate decomposition and observation method}

The recovered and accumulated hydrate was decomposed by the heating or depressurization method without flowing water. The decomposition behavior of the hydrate sedimentary layer and the generated bubbles above the deposit were taken continuously with a digital camera. Figure 2 shows the decomposition of the hydrate deposit and the rising bubbles. The captured image was analyzed using image analysis software (ImageJ 1.50i, developed by National Institutes of Health, USA). The bubble rising velocity was determined by combining the continuously captured images and measuring the moving distance. Since the water was sufficiently saturated with the gas component during decomposition, the bubble radius reduction by gas dissolution was negligible.

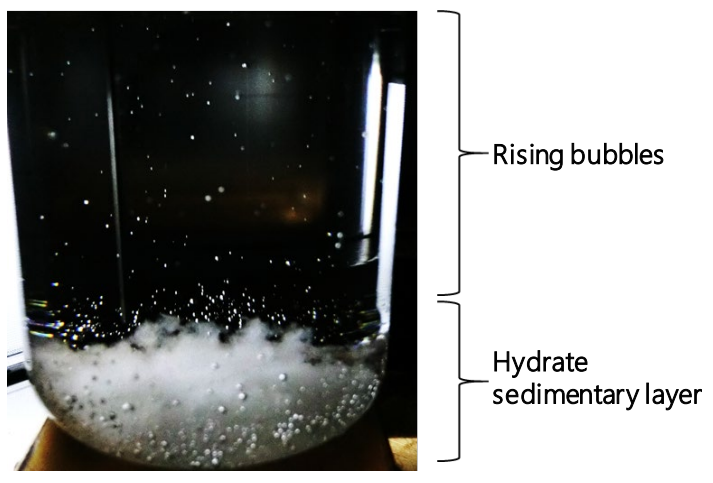

Figure 2. Decomposition appearance of hydrate sedimentary layer

\section{Results and Discussion}

\subsection{Bubble rising velocity}

Although most of rising bubbles look like a sphere the bubble radius $r_{\mathrm{e}}$ is expressed by Equation 1. Symbols $a$ and $b$ are the major and minor axes of the bubble.

$$
r_{e}=\sqrt[3]{a^{2} b}
$$

\subsubsection{Navier-Stokes equation}

The resistance coefficient $C_{D}$ (Equation 2) and the rising velocity $U$ (Equation 3 ) derived from the Navier-Stokes equation as the bubble Reynolds number $R e>1$ were used as the theoretical rising velocity values (Chemical Engineering Association, 1969).

$$
\begin{gathered}
C_{D}=\frac{48}{R e} \\
U=\frac{\rho g d^{2}}{36 \mu}
\end{gathered}
$$




\subsubsection{Presence or absence of hydrate film}

In addition to the Navier-Stokes equation, the theoretical value of the rising velocity was derived depending on the bubble shape and the presence of the hydrate film according to available literature (Bigalke et al., 2010). The rising velocity $U_{\mathrm{T}}$ is determined by the balance of buoyant and friction forces.

$$
U_{T}=\sqrt{\frac{8 g r_{e}\left(\rho-\rho_{p}\right)}{3 C_{D} \rho}}
$$

If $C_{\mathrm{D}}=48 / R e$, this is equal to Equation 2. The coefficient of resistance $C_{\mathrm{D}}$ is determined by the friction coefficients $f_{i}, r_{e}$ and $a$ (Equation 5). The term $\left(a / r_{e}\right)^{2}$ is called the deformation factor. Subscripts $h$ and $c$ represent the presence or absence of a hydrate film, respectively.

$$
C_{D}=f_{c, h}\left(\frac{a}{r_{e}}\right)^{2}
$$

The coefficient of friction $f_{c}$ of bubbles without a hydrate film is expressed by Equation $6 \mathrm{a}$, and the coefficient of friction $f_{\mathrm{c}}$ of the covered bubble with hydrate film is expressed by Equation $6 \mathrm{~b} . E_{\mathrm{o}}$ is a dimensionless number representing the ratio of buoyancy to surface tension.

$$
\begin{aligned}
& f_{c}=\frac{48}{R e}+0.9 \frac{0.75 E_{O}{ }^{2}+0.6}{0.75 E_{O}{ }^{2}+14.5} \\
& f_{h}=\frac{9}{\sqrt{R e}}+0.9 \frac{0.75 E_{O}{ }^{2}}{0.75 E_{O}{ }^{2}+4.5}
\end{aligned}
$$
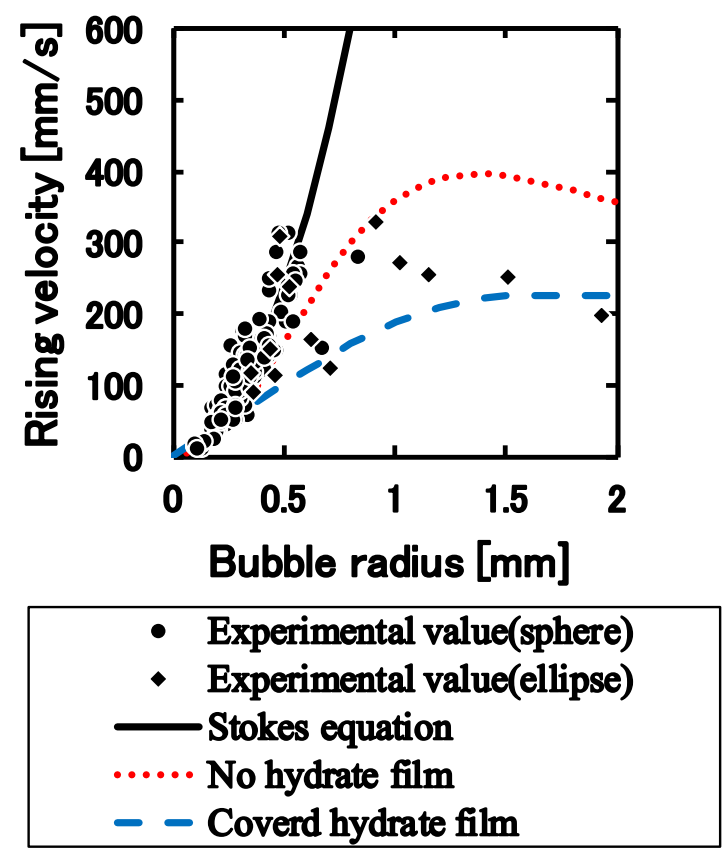

Figure 3 Relationship between bubble radius and rising velocity $(0.2 \mathrm{MPa})$

Figure 3 shows the relationship between the bubble radius and the rising velocity under the decomposition conditions $(0.2 \mathrm{MPa}, 288 \mathrm{~K})$. The rising velocity of small spherical bubbles with a radius of about $0.5 \mathrm{~mm}$ or less corresponded to the theoretical curve by the NavierStokes (closed circles). Although it does not agree with the theoretical value of no hydrate film curve (dotted), this may be due to a difference in the influence of the bubble shape considered in the theoretical equation. The relatively large elliptical bubble (closed diamonds) showed a behavior close to the theoretical rising velocity value with hydrate film (dashed curve). As a result, it is possible that the elliptical bubble may behave like a rigid body, for example due to the coating of a hydrate film or the like, when rising. The hydrate deposit container temperature was constant between 1 hour and 1 hour and a half from the start of heating, and similar results were observed at any period.

The same behavior was shown for smaller bubbles regardless of the pressure condition (Figures 4 for 0.1 $\mathrm{MPa}$ and 5 for $0.3 \mathrm{MPa}$ ). In these conditions, unfortunately, there is no data of large bubbles including the ellipse bubble because large bubble generation occurred sporadically and we couldn't take the images of it adequately. The larger bubble behavior needs to be confirmed in further investigation.

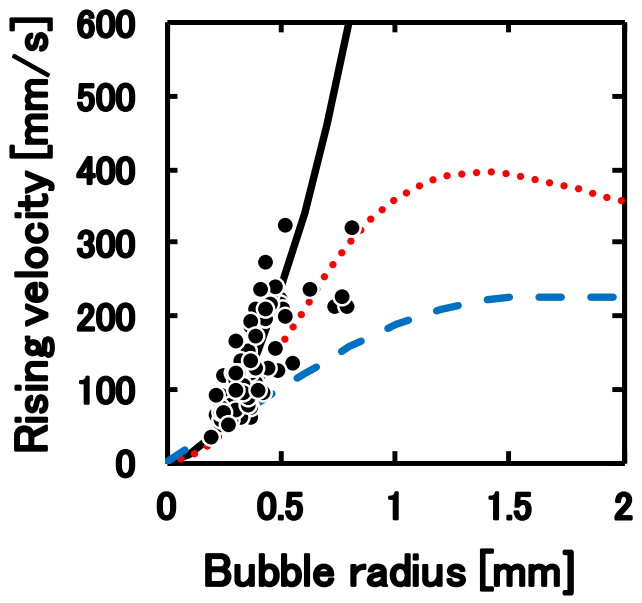

Figure 4 Relationship between bubble radius and rising velocity $(0.1 \mathrm{MPa})$

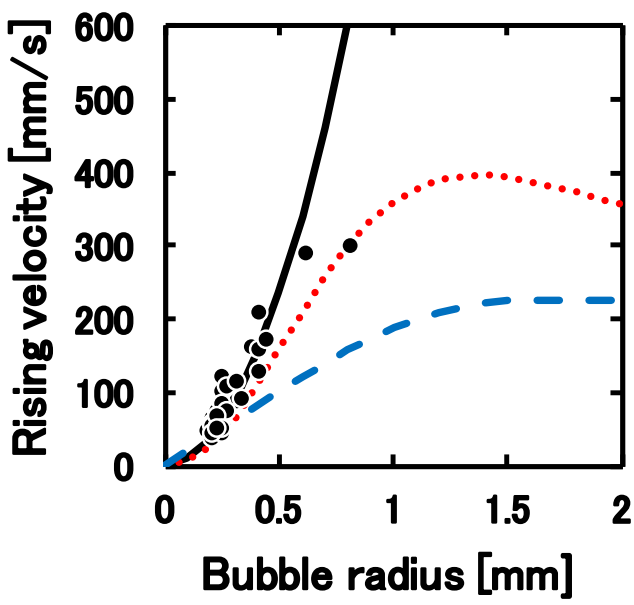

Figure 5 Relationship between bubble radius and rising velocity $(0.3 \mathrm{MPa})$

3.2 Conditions closer to the hydrate equilibrium 


\subsubsection{Heating method}

Hydrate decomposition was carried out under the pressure and temperature conditions closer to the hydrate equilibrium curve. Although no bubbles were observed by sight under decomposition conditions $(0.3 \mathrm{MPa}$, $286 \mathrm{~K})$ by the heating method, it was confirmed that the apparent thickness of the deposited layer decreased slowly. The fine bubbles that are too small might be generated. To understand the situation in the hydrate sedimentary vessel, how heat is transmitted to the hydrate sedimentary vessel by the presence or absence of hydrate is compared by Equation 7.

$$
-\ln \frac{(T s-T)}{(T s-T 0)}=k t
$$

where $T_{\mathrm{s}}$ is the temperature set in the thermal chamber, $T_{0}$ and $T$ are the initial temperature and the temperature at time in hydrate sedimentary vessel, respectively. Figure 6 shows the temperature change in the hydrate sedimentary vessel according to Equation 7 . The slope of the temperature change varied apparently in stages. In the presence of hydrate, the slope varied firstly at approximately 100 seconds at which the temperature in the vessel reached the hydrate equilibrium temperature $282 \mathrm{~K}$. This may imply that the endothermic hydrate decomposition and the bubble generation make it difficult to heat the vessel inside than the water-only condition. Further investigation is required for the effect of hydrate decomposition and bubble generation.

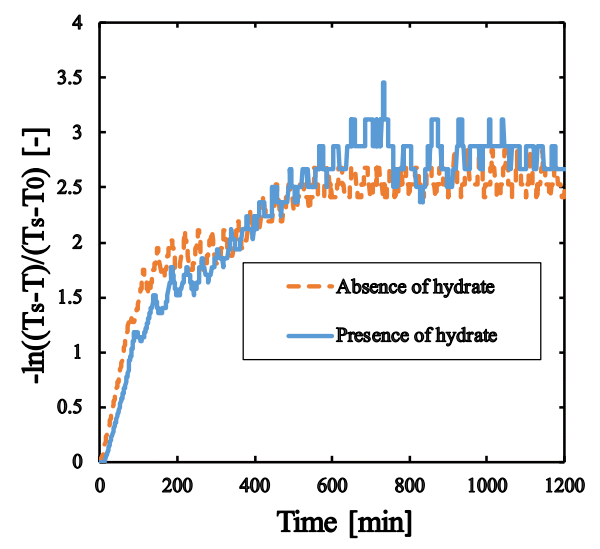

Figure 6. Temperature change in the hydrate sedimentary vessel (heating method)

\subsubsection{Depressurization method}

Next, the hydrate decomposition was carried out by the pressure reduction method under the condition of 0.1 $\mathrm{MPa}$ and $280 \mathrm{~K}$. After hydrate formation, only pressure was reduced to atmosphere. Figure 7 shows the temperature change in the vessel during the depressurized hydrate decomposition. The temperature in the hydrate sedimentary vessel lowered below the initial temperature. In this case, the bubble generation was not observed visually. Although the R22 hydrate equilibrium temperature is about $274 \mathrm{~K}$ at $0.1 \mathrm{MPa}$, it is necessary to consider the possibility of localized temperature reduction and rehydration due to the endothermic decomposition of the hydrate.

\section{Conclusions}

We investigated the hydrate deposit decomposition behavior at various temperature and pressure conditions, heating method and depressurization method.

(1) The experimental rising velocities of small spherical bubbles agreed well with the theoretical value by the Navier-Stokes equation. The relatively large elliptical bubbles showed a behavior close to the theoretical value of bubble with hydrate film. The rising velocity may decrease for any reason; for example use of saturated water. Further investigation is needed for the influence of the bubble shape and hydrate film terms in the theoretical equation.

(2) Under the pressure and temperature conditions closer to the hydrate equilibrium, almost no generated bubbles could be identified visually. This may be because of the fine bubble generation.

In addition, we will investigate the influence on the bubble behavior when using unsaturated aqueous solution or mixed gas. Ultimately, the goal is to bring it closer to seafloor conditions in the presence of methane hydrate.

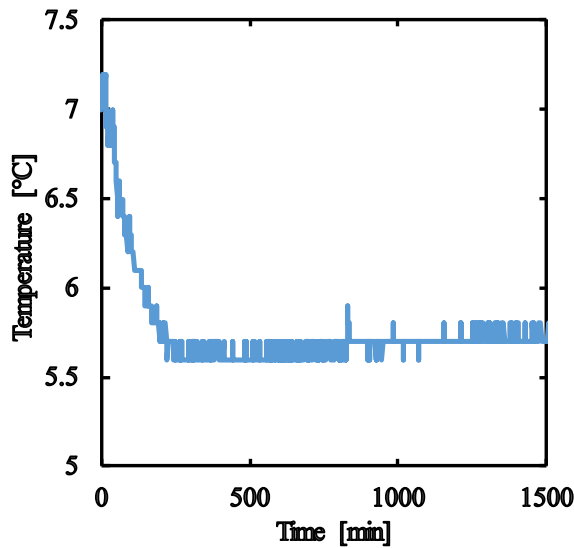

Figure 7. Temperature change in the hydrate sedimentary vessel (Depressurization method)

\section{Acknowledgements}

This research is funded by JSPS Grants-in-Aid for Scientific Research 16K06821.

\section{References}

Aoyama, C., K. Tani, H. Fukuoka, H. Watanabe, and A. Kawagishi; "Inspection of Recovery Technology Using Dome-Shaped Membrane Structure," The 9th Comprehensive Symposium on Methane Hydrate (CSMH-9), B-13 (2017)

Bigalke, N. K., L. I. Enstadb, G. Rehderca, and G. Alendald; "Terminal Velocities of Pure and Hydrate 
Coated $\mathrm{CO}_{2}$ Droplets and $\mathrm{CH}_{4}$ Bubbles Rising in a Simulated Oceanic Environment," Deep Sea Research Part I: Oceanographic Research Papers 57, 1102-1110 (2010)

Chemical Engineering Association; "Advances in Chemical Engineering Vol.3 Bubble and Droplet Engineering," Nikkan Kogyo Shimbun (1969)

Kataoka, S., A. Yamashita, N. Minami, S. Nishio, T. Anbe, Y. Yokoyama, M. Hyodo, and M. Grachev; "Geotechnical Properties of the Shallow Type Methane Hydrate-Bearing Sediments in the Lake Baikal" Japanese Geotechnical J., 2 (2), 95-105 (2007)

Sato M., S. Nagakubo, T. Uchida, and A. Tani; "Methane Hydrate II Origin of Methane Hydrate, Exploration and Resource" J. Japan Inst. Energy, 95 (7), 572-585 (2016) 